\title{
Single and Multiple Phase Shifts Tilted Fiber Bragg Gratings
}

\author{
Andrea Cusano, ${ }^{1}$ Domenico Paladino, ${ }^{1}$ Agostino Iadicicco, ${ }^{2}$ \\ Stefania Campopiano, ${ }^{2}$ and Christophe Caucheteur ${ }^{3}$ \\ ${ }^{1}$ Optoelectronic Division, Engineering Department, University of Sannio, Corso Garibaldi 107, 82100 Benevento, Italy \\ ${ }^{2}$ Department for Technologies, University of Naples Parthenope, Centro Direzionale, Isola C4, 80143 Napoli, Italy \\ ${ }^{3}$ Electromagnetism and Telecommunication Unit, Faculty of Engineering Mons, 7000 Mons, Belgium
}

Correspondence should be addressed to Andrea Cusano, a.cusano@unisannio.it

Received 13 November 2008; Accepted 12 February 2009

Recommended by Wojtek J. Bock

\begin{abstract}
The spectral behavior of single and multiple phase shifts tilted fiber Bragg gratings has been experimentally investigated. To this aim, a simple and cost-effective postprocessing technique based on local thermal treatment was used to create arbitrary phase shifts along the tilted grating structure. In particular, UV written tilted fiber Bragg gratings were treated by the electric arc discharge to erase the refractive index modulation in well-defined regions. We demonstrate that these defects give rise to interference pattern for all modes, and thus defect states can be achieved within all the attenuation bands, enabling a simple wavelength independent spectral tailoring of this class of devices.
\end{abstract}

Copyright (c) 2009 Andrea Cusano et al. This is an open access article distributed under the Creative Commons Attribution License, which permits unrestricted use, distribution, and reproduction in any medium, provided the original work is properly cited.

\section{Introduction}

In the last decades, intense research on fiber Bragg gratings (FBGs) as strain and temperature sensing elements has been conducted: however, in order to increase the applicability of this technology, new research paths have been opened. One of them consists in studying structures far more complex than the widely used uniform FBG. Among these, structures such as long-period grating [1], chirped FBG [2], and tilted FBG (TFBG) [3] have been proposed for various sensing applications. In particular, TFBGs have been recently investigated for various sensing applications ranging form external refractive index, bending, and many others $[4,5]$, providing an exciting technological platform to be employed in many industrial sectors. In order to enlarge the potentialities of this class of devices, here, we investigate the spectral behavior of single and multiple phase shifts TFBGs fabricated by a low cost and simple postprocessing technique based on local heat treatment. Different from common phase shift gratings [6], here the electric arc discharge (EAD) was used as postprocessing technique to locally erase the grating modulation, producing single and multiple phase shifts without phase masks or patterned coatings [7] independently on the operating optical wavelength [8].
For the first time to the best of our knowledge, we report on the spectral characteristics in weakly TFBG with singleand multiphase shifts focusing the attention on the capability of the proposed method to reshape and to force defect states in all attenuation bands.

\section{Experiment}

The investigated TFBG has been manufactured into hydrogen-loaded Corning single-mode fiber by means of frequency-doubled Argon ion laser emitting at $244 \mathrm{~nm}$ [9]. A $1060 \mathrm{~nm}$ uniform phase mask was mounted on a rotating stage in order to apply the desired tilt. The transmitted spectrum of a $1 \mathrm{~cm}$ long $3^{\circ}$ TFBG measured in air is shown in Figure 1. TFBGs belong to the short-period grating family (period of the refractive index modulation $\sim 0.5 \mu \mathrm{m}$ ), and thus their transmitted spectra exhibit an attenuation band related to the coupling with the contrapropagating core mode (i.e., the Bragg peak at $1539.665 \mathrm{~nm}$ ). However, since the index modulation pattern is blazed with respect to the fiber axis, the coupling to circularly and noncircularly symmetric cladding modes is enhanced while the selfbackward coupling of the core mode is reduced as compared 
with uniform FBGs. According to TFBG theory, discrete resonance bands, related to the coupling of discrete bounded cladding modes, are achieved for wavelengths shorter than the Bragg resonance (see Figure 1, cladding mode dips are observed for wavelengths shorter than $1537.100 \mathrm{~nm}$ ) [3-9]. The resonance wavelengths depend on the effective refractive indices of core and cladding modes and on the grating pitch. An important feature of the weakly tilted FBGs is the presence of a strong "ghost mode" resonance, immediately to the left of the Bragg resonance. In Figure 1, it can be observed at $1537.365 \mathrm{~nm}$. The ghost resonance corresponds to a group of low-order asymmetric cladding modes which are well confined in the core and less sensitive to the claddingexternal medium interface [5].

EAD technique has been already demonstrated to be a valid low-cost postprocessing tool to induce localized defects along uniform FBGs for their photonic bandgap engineering [8]. EAD stage locally erases a well-defined region of the grating creating a distributed phaseshift or equivalently a Fabry-Perot like structure [8]. In this work, EAD approach, carried out by a commercial fusion splicer unit (Fujikura FSM-50S), was applied to TFBGs for the first time to our knowledge. Experimental training carried out on standard communication fiber allowed us to determine the optimum processing parameters. As a result, fusion current and arc duration were manually selected to $17.1 \mathrm{~mA}$ and 100 milliseconds, respectively, in order to locally erase the grating structure while keeping the optical fiber integrity.

To investigate the effect of single- and multiphase shifts along TFBGs, EAD treatment was repeated at various precise locations along the grating length as schematized in Figure 2. The first EAD was applied in the grating center, while other EADs were forced at $3 / 4$ and $1 / 4$ of the grating length. To this aim, the grating area was localized with $1 \mathrm{~mm}$ resolution by observing the radiated light when the grating is illuminated by red light.

TFBG transmitted spectra were recorded at each EAD step by a simple optoelectronic setup with a wavelength resolution of $1 \mathrm{pm}$ involving a tunable laser source (Ando AQ4321D) and an optical spectrum analyzer (Ando AQ6317C).

\section{Results}

In this section, the effects of single and multiple phase shifts created by successive EAD steps are investigated with regards to all the coupling mechanisms exhibited by TFBGs.

Figure 3 compares the transmitted spectra of the pristine grating (black solid line) and the structure with a single phase shift (dashed line). The attention was first focused on the attenuation bands related to cladding modes bounded at the cladding-air interface. The specific wavelength range was chosen in correspondence of the deepest attenuation bands (high-order cladding modes). Here, attenuation bands related to azimuthally (dips of about $-39 \mathrm{~dB}$ ) and nonazimuthally (dips of about $-29 \mathrm{~dB}$ ) symmetric cladding modes can be observed. As evident, a single phase shift is able to produce the formation of an allowed band or

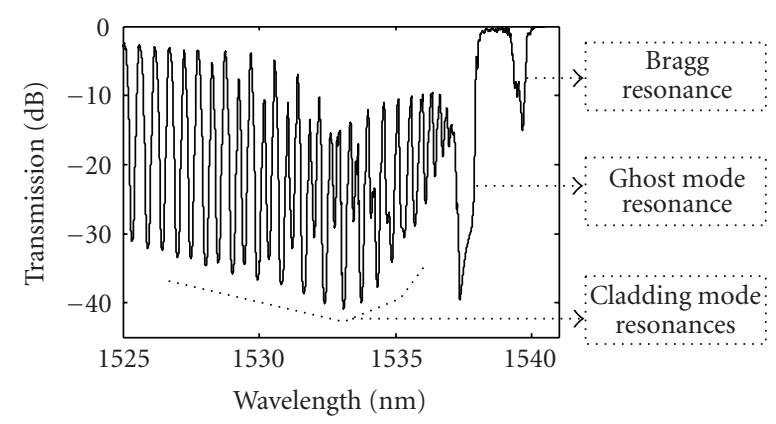

FIgUre 1: Transmission spectrum of the utilized $3^{\circ}$ TFBG.

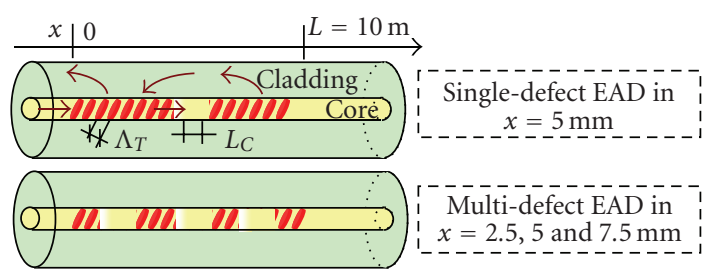

FIGURE 2: Schematic diagram of the experimentally realized singleand multidefect TFBG (not in scale).

defect state inside the attenuation band associated to each cladding modes. This means that the local grating erasure forms an interferometric structure for cladding modes. The principle of operation can be explained as follows from Figure 2. The TFBG section located at the right of the phase shift region couples light at specific wavelengths toward contrapropagating cladding modes. These cladding modes delayed by the phase shift region reach the first TFBG section where recoupling toward the core mode occurs. A continuous interference can thus be observed due to the interaction between the direct core mode and part of core mode due to cladding modes recoupling. The main effect is the creation of attenuation bands characterized by a single and narrow range of allowed optical wavelengths. Its location depends on the phase shift amount and thus on the erased region length and the effective index of the cladding modes. Besides the defect state formation, the EAD stage significantly affects the depth of all attenuation bands too. In the investigated spectral range (see Figure 3), attenuation bands depth related to azimuthally symmetric cladding modes passes from $-39 \mathrm{~dB}$ to $-33 \mathrm{~dB}$; whereas those related to nonazimuthally symmetric modes decrease of about $7 \mathrm{~dB}$.

The spectrum of a multidefect structure is also plotted in Figure 3 (grey solid curve). The multiphase shift mechanism significantly reshapes the grating spectrum. The new coupling regime, in fact, leads to well-defined optical wavelengths which in turn are able to propagate without significant losses when compared with the pristine structure. Moreover, for each attenuation band, we can observe the formation of two weak dips on both sides of the pristine symmetric and asymmetric resonance bands characterized by a depth of approximately $15 \mathrm{~dB}$. As matter of fact, the 


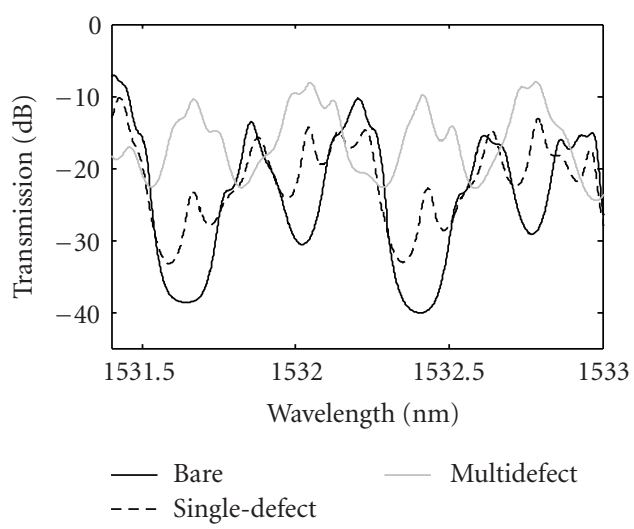

Figure 3: Spectra of the pristine, single-defect, and multidefect TFBG in the wavelength range involving few cladding modes attenuation bands.

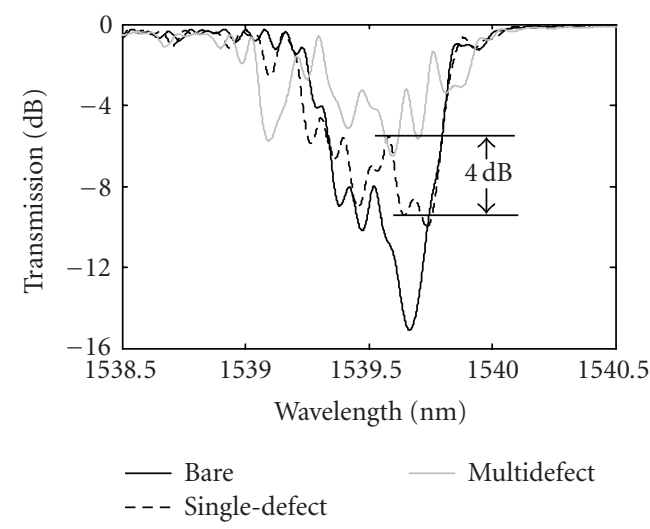

Figure 4: Spectra of the pristine, single-defect, and multidefect TFBG in the wavelength range involving the Bragg attenuation band.

multi-EAD treatment forces a drastic peak-to-peak amplitude decrease from approximately $30 \mathrm{~dB}$ (pristine grating) to $15 \mathrm{~dB}$ in the investigated spectral range. However, we believe that the spectral location and the amplitude of new dips can be manipulated by acting on the phase shift amount of each defect and defect position along the grating. Hence, from these preliminary results, EAD represents a simple tool enabling complete photonic bandgap engineering with regards to the cladding mode response in UV-written TFBGs.

Figure 4 reports the same analysis as Figure 3 focusing this time the attention on the Bragg resonance. The spectrum of the grating subjected to single EAD clearly shows the formation of a $4 \mathrm{~dB}$ deep defect state at $1539.581 \mathrm{~nm}$ inside the Bragg band; whereas the modulation on the short wavelength side was already present in the unperturbed spectrum. The grating erasure, in fact, acts as a FabryPerot cavity for the core mode reflected from the two TFBG sections. This effect has been widely investigated and reported elsewhere [8].

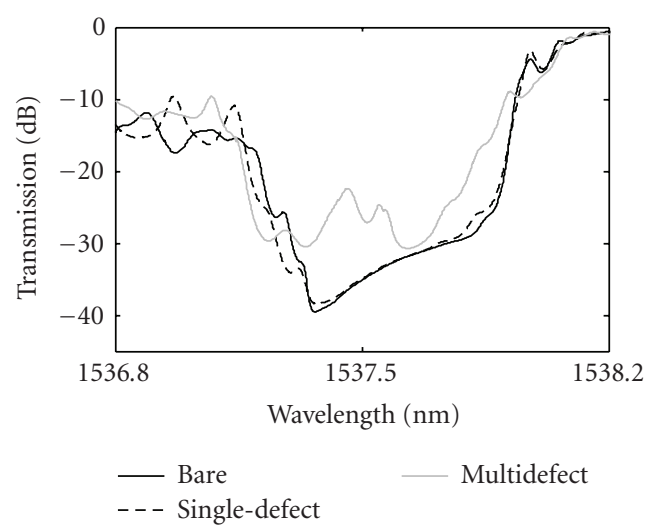

FIgure 5: Spectra of the pristine, single-defect, and multidefect TFBG in the wavelength range involving the ghost band.

Additionally the introduction of multiphase shift along the grating structure decreases the strength of the unperturbed grating mirrors and induces a multi-interference regime. The former effect is responsible for a significant amplitude decrease (the band depth decreases of $8.6 \mathrm{~dB}$ ) and bandwidth enlargement with consequent loss in wavelength selectivity. On the contrary, multibeating regime forces the formation of multiple fringes inside the original Bragg stopband.

Finally, Figure 5 shows the spectral effects due to successive EAD operations on the attenuation band related to the so-called "ghost mode." Here, we observe a negligible effect on the spectral shape of the ghost band during the first two EAD steps. Finally, we observe the formation of allowed bands also for the ghost band when the third EAD was created.

In particular, two allowed bands of $8 \mathrm{~dB}$ and $6 \mathrm{~dB}$ at $1537.459 \mathrm{~nm}$ and $1537.549 \mathrm{~nm}$, respectively, can be clearly remarked. The presence of a more complex allowed bandwidth within the ghost band could be explained by recognizing from [5] that this attenuation band is related not to a single but to a group of cladding modes well confined in the fiber core. The leaky nature of the ghost modes at the core cladding interface and the spatial location of the phase shift section able to produce a defect state modification in the ghost band are actually under investigation to a full comprehension of the phase shift mechanism in this case.

On the base of these results, the proposed method seems to work correctly as low cost, postprocessing, and wavelength independent tool capable to completely reshape the spectrum of weakly TFBGs. However, it is reasonable to believe that spectral signature can be ad hoc tailored by acting on number, distribution, and size of the heat treated regions with benefices in practical applications. For instance, finer spectral features lead to advantages in terms of lower resolution when the device is interrogated by using wavelength shift measurements. The core/cladding mode recoupling and the consequent interefernce regime, instead, could be exploited in new configurations to enhance the sensing performances of this class of devices. For instance, for chemical application where the small changes in the 
surrounding refractive index need to be monitored, the defect states should exhibit a relative shift inside each cladding mode band with consequence enhancement in sensitivity against the refractive index as compared to the cladding mode dips shifts. However, experimental investigations on this topic are currently in progress. Additional, the core/cladding mode recoupling regime due to localized perturbations [8] could be involved to improve the performance of new sensing scheme based on reflected light monitoring $[5]$.

Also, spectral features tailoring could enable new filtering functionalities for communication applications.

\section{Conclusion}

The spectral characteristics of single- and multiphase shift weakly tilted FBG have been investigated. EAD was used as low-cost tool to achieve local and distributed phase shift sections along the grating. Spectral modifications involving cladding modes dips, the Bragg band, and also the ghost resonance have been observed, demonstrating the possibility to tailor the spectra of this class of devices by using simple postprocessing and wavelength independent fabrication. On the basis of these results, we believe that playing on defects number and shape, their longitudinal positioning, new all fiber photonic devices can be achieved and optimized for specific applications.

\section{References}

[1] X. Shu, L. Zhang, and I. Bennion, "Sensitivity characteristics of long-period fiber gratings," Journal of Lightwave Technology, vol. 20, no. 2, pp. 255-266, 2002.

[2] M. G. Xu, L. Dong, L. Reekie, J. A. Tucknott, and J. L. Cruz, "Temperature-independent strain sensor using a chirped Bragg grating in a tapered optical fibre," Electronics Letters, vol. 31, no. 10, pp. 823-825, 1995.

[3] G. Laffont and P. Ferdinand, "Tilted short-period fiber-Bragggrating-induced coupling to cladding modes for accurate refractometry," Measurement Science and Technology, vol. 12, no. 7, pp. 765-770, 2001.

[4] D. Paladino, A. Cusano, P. Pilla, S. Campopiano, C. Caucheteur, and P. Mégret, "Spectral behavior in nano-coated tilted fiber Bragg gratings: effect of thickness and external refractive index," IEEE Photonics Technology Letters, vol. 19, no. 24, pp. 20512053, 2007.

[5] T. Guo, A. Ivanov, G. Chen, and J. Albert, "Temperatureindependent tilted fiber grating vibration sensor based on cladding-core recoupling," Optics Letters, vol. 33, no. 9, pp. 1004-1006, 2008.

[6] R. Kashyap, P. F. McKee, and D. Armes, "UV written reflection grating structures in photosensitive optical fibres using phaseshifted phase masks," Electronics Letters, vol. 30, no. 23, pp. 1977-1978, 1994.

[7] A. Cusano, D. Paladino, A. Cutolo, I. Del Villar, I. R. Matias, and F. J. Arregui, "Spectral characteristics in long-period fiber gratings with nonuniform symmetrically ring shaped coatings," Applied Physics Letters, vol. 90, no. 14, Article ID 141105, 3 pages, 2007.

[8] A. Cusano, A. Iadicicco, D. Paladino, S. Campopiano, and A. Cutolo, "Photonic band-gap engineering in UV fiber gratings by the arc discharge technique," Optics Express, vol. 16, no. 20, pp. 15332-15342, 2008.

[9] C. Caucheteur and P. Mégret, "Demodulation technique for weakly tilted fiber Bragg grating refractometer," IEEE Photonics Technology Letters, vol. 17, no. 12, pp. 2703-2705, 2005. 

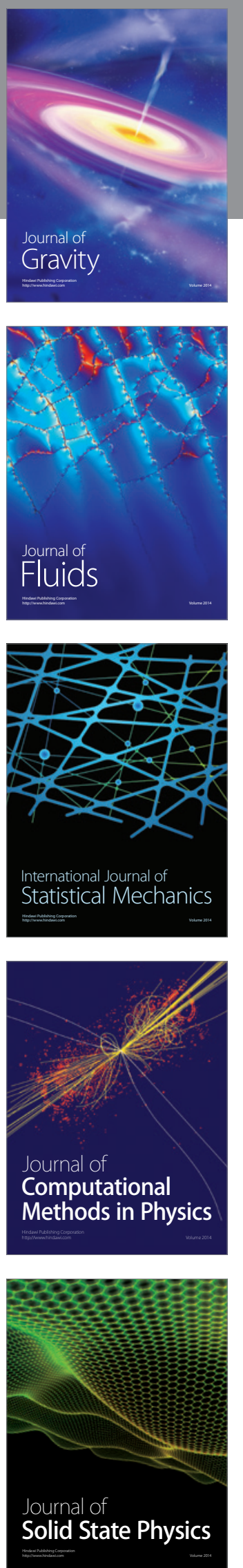

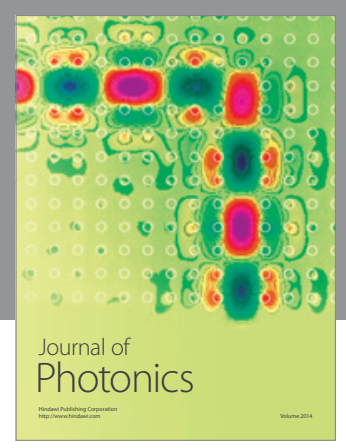

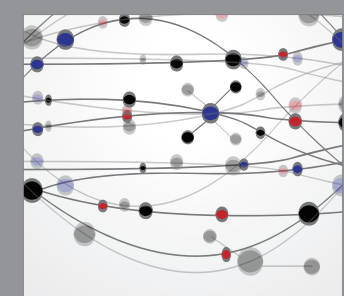

The Scientific World Journal
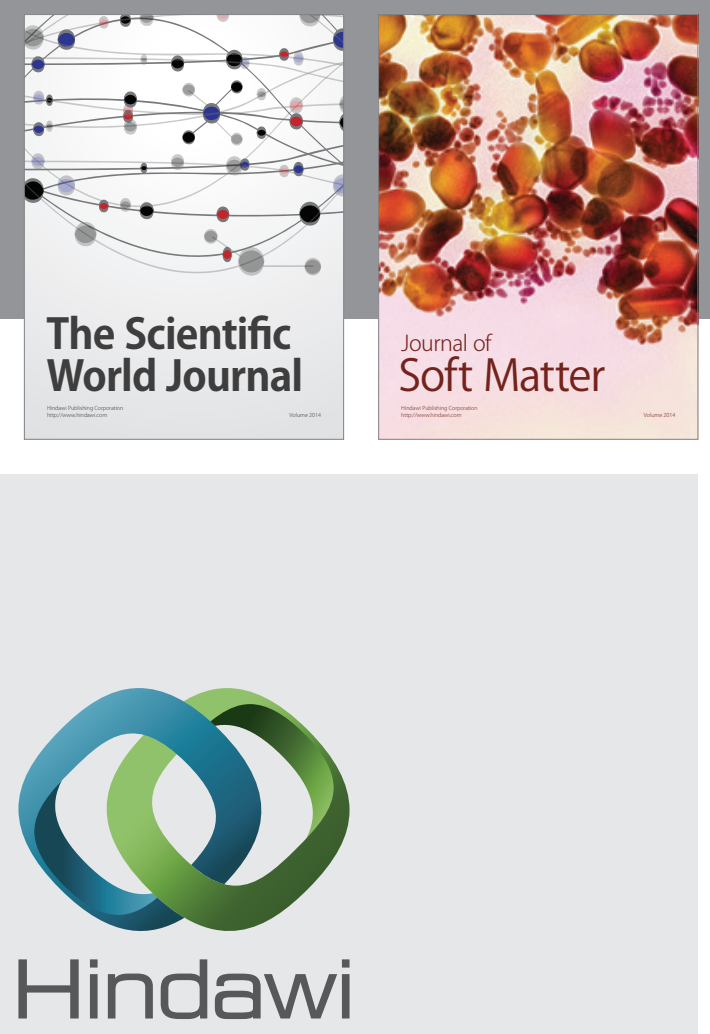

Submit your manuscripts at

http://www.hindawi.com

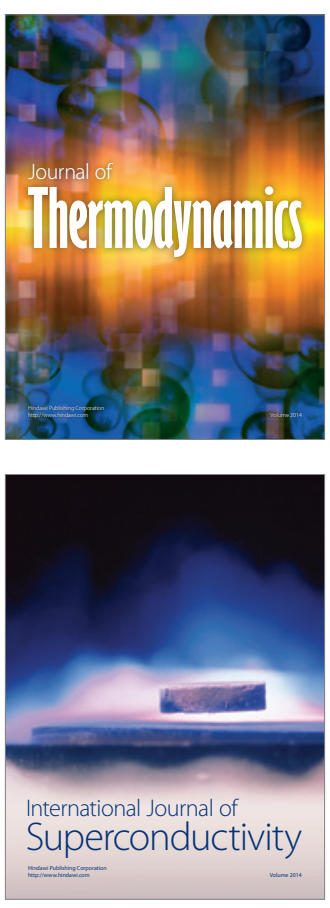

